\title{
The inhomogeneous wind of the LBV candidate Cyg OB2 No.12
}

\author{
Valentina G. Klochkova ${ }^{1}$, Eugene L. Chentsov ${ }^{1}$ and Anatoly S. \\ Miroshnichenko ${ }^{2}$ \\ ${ }^{1}$ Special Astrophysical Observatory RAS, Nizhnij Arkhyz, 369167, Russia \\ email: valenta@sao.ru, echen@sao.ru \\ ${ }^{2}$ Dept. of Physics \& Astronomy, University of North Carolina at Greensboro, \\ P.O. Box 26170, Greensboro, NC 27402-6170, USA; email: a-mirosh@uncg.edu
}

\begin{abstract}
We present the results of high-resolution spectroscopy of the extremely luminous star Cyg OB2 No. 12. We identified about 200 spectral features in the range 4552-7939 $\AA$, including the interstellar $\mathrm{NaI}$, K I lines and numerous very strong DIBs, along with the He I, C II, and Si II lines. An MK spectral type we derived for the object is $\mathrm{B} 4.5 \pm 0.5 \mathrm{Ia}^{+}$. Our analysis of the radial velocity data shows the presence of a gradient in the stellar atmosphere, caused by both atmospheric expansion and matter infall onto the star. The $\mathrm{H} \alpha$ emission displays broad Thompson wings, a slightly blue-shifted P Cyg type absorption component and a time-variable core absorption. We conclude that the wind is variable in time.
\end{abstract}

Keywords. stars: supergiants, stars: circumstellar matter

\section{Introduction}

For stars in clusters the evolutionary stage, age, and luminosity can be determined more reliably, whereas they are rather uncertain for field stars. It is especially important to study group members that are rare, such as LBV-stars. From this point of view, young Cyg OB2 association is of special interest. Many unevolved O/Of-stars have been identified there as well as an LBV candidate - the variable star No. 12. Its luminosity is $\log \mathrm{L} / \mathrm{L} \odot=6.26$ (de Jager 1998) at the association distance of $1.7 \mathrm{kpc}$.

\section{Observations and results}

Optical spectra of Cyg OB2 No. 12 were taken using the échelle spectrographs of the 6-meter telescope of the Special Astrophysical Observatory. On June 12, 2001, we used the PFES spectrograph (Panchuk et al. 1997) with a $1040 \times 1170$-pixel CCD at the prime focus and got a spectrum with a resolution of $R=\lambda / \Delta \lambda \sim 15000\left(20 \mathrm{~km} \mathrm{~s}^{-1}\right)$. Later we used the NES spectrograph (Panchuk et al. 2009) equipped with a $2048 \times 2048$-pixel CCD and an image slicer and obtained spectra with $R \sim 60000\left(5 \mathrm{~km} \mathrm{~s}^{-1}\right)$ on April 12, 2003 and on December 8, 2006.

The spectral types we derived for three dates were the same within the errors: B5.0 \pm 0.5 , $\mathrm{B} 4.8 \pm 0.5$ and $\mathrm{B} 4.0 \pm 0.5$. The luminosity type is $\mathrm{Ia}^{+}$. The high luminosity is supported by the strong O I $7773 \AA$ IR-triplet whose equivalent width of $1.14 \AA$ corresponds to an absolute visual magnitude of $\mathrm{M}_{V}<8$ mag.

The radial velocities $\left(\mathrm{V}_{\mathrm{r}}\right)$ measured from the absorption line cores vary with time and with the line intensity. The weakest lines give $\mathrm{V}_{\mathrm{r}}$ lower than $\mathrm{V}_{\mathrm{sys}}=-11 \mathrm{kms}^{-1}$ (Klochkova \& Chentsov 2004) by 5, 14 and $15 \mathrm{~km} \mathrm{~s}^{-1}$ in 2001, 2003, 2006, respectively, suggesting a variable expansion rate of the layers where they form. The left panel of 

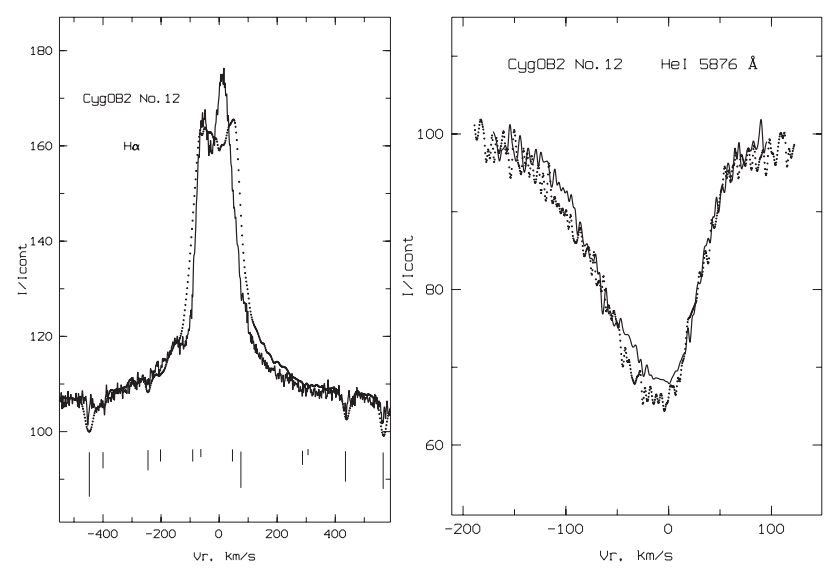

Figure 1. H $\alpha$ (left) and He I $5876 \AA$ (right) profiles in the spectra of Cyg OB2 No. 12 obtained on June 12, 2001 (dotted line) and April 12, 2003 (solid line). The absorption features seen within the $\mathrm{H} \alpha$ core correspond to transitions in the line rather than to the telluric spectrum, whose contribution was carefully removed. The vertical dashes show the positions of telluric lines, with the dash lengths proportional to the line strength.

Fig. 1 shows that the $\mathrm{H} \alpha$ profile varies with time, but its principal features are preserved: a strong bell-shaped emission, with a dip at the short-wavelength slope, a sheared peak, and extended Thompson wings. The blue-shifted absorption is barely visible in June 2001 and is more pronounced in April 2003, but can be traced at least to $\mathrm{V}_{\mathrm{r}}=-160 \mathrm{~km} \mathrm{~s}^{-1}$ in both cases; i.e., to the same limit that is reached by the blue wings of the absorption lines of Si II and HeI (the latter shown in the right panel of Fig. 1).

The wind terminal velocity is $\sim 150 \mathrm{~km} \mathrm{~s}^{-1}$. The intensity inversions in the upper part of the $\mathrm{H} \alpha$ profile indicate that the wind is not uniform. In addition to the high velocity material mentioned above, it contains a fair amount of material that is nearly stationary relative to the star or is even falling onto the stellar surface. Coexistence of lines with direct and inverse P Cygni profiles in the same spectrum, and even combinations of such features in the profile of the same line leads us to reject a spherical symmetry wind. It is possible that the slow part of the wind also contributes to the absorption profiles. So far, this possibility is supported by the coincident velocities for the central dips of the $\mathrm{H} \alpha$ line and the well-formed cores of strong absorption lines (He I 5876 $\AA$ in 2001 and Si II $6347 \AA$ in 2003), as well as by the fact that the blue shift of all the absorption lines in the 2003 spectrum relative to their positions in 2001 was accompanied by a similar shift of the central dip in $\mathrm{H} \alpha$. At any rate, both the hydrogen lines and the strongest absorption lines in the visual spectrum Cyg OB2 No. 12 are partially formed in the wind.

\section{Acknowledgements}

This research was supported by the Russian Foundation for Basic Research (project no. 08-02-00072 a).

\section{References}

de Jager, C. 1998, A\&AR, 8, 145

Klochkova, V. G. \& Chentsov, E. L. 2004, Astron. Rep., 48, 1005

Panchuk, V. E., Najdenov, I. D., Klochkova, V. G., Ivanchik, A. B. et al. 1997, Bull. Special Astrophys. Obs., 44, 127

Panchuk, V. E., Klochkova, V. G., Yushkin, M.V., \& Naidenov, I. D. 2009, Opticheskii Zhurn., 76,42 